\title{
The CAT-262 C>T, MnSOD Ala16Val, GPX1 Pro198Leu Polymorphisms Related to Oxidative Stress and the Presence of Gastric Lesions
}

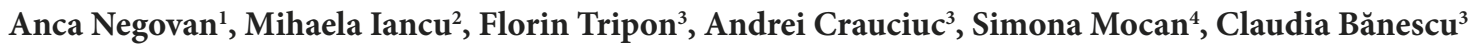

1) University of Medicine,

Pharmacy, Science and

Technology of Târgu Mureș

Clinical Science-Internal

Medicine, Tirgu Mureș;

2) Iuliu Hatieganu University

of Medicine and Pharmacy,

Department of Medical

Informatics and Biostatistics,

Cluj-Napoca;

3) Genetics Laboratory, Center

for Advanced Medical and

Pharmaceutical Research,

UUniversity of Medicine,

Pharmacy, Science and

Technology of Târgu Mureș;

4) Emergency County

Hospital, Pathological

Department, Târgu Mureș

Romania

\author{
Address for correspondence: \\ Mihaela Iancu \\ Iuliu Hatieganu University of \\ Medicine and Pharmacy \\ Department of Medical \\ Informatics and Biostatistics, \\ Louis Pasteur St, no. 6, 400349 \\ Cluj-Napoca, Romania, \\ mmihaela.iancu@yahoo.com
}

Received: 17.07 .2018

Accepted: 17.11.2018

\begin{abstract}
Background \& Aims: The increased oxidative stress plays an important role in gastro-duodenal ulcers and gastric cancer occurrence. We investigated the association between the genetic polymorphisms of genes encoding the antioxidative enzymes CAT, GPX and SOD and the occurrence of gastric lesions, considering also the environmental risk factors such as $\mathrm{H}$. pylori infection, drug exposure, smoking and alcohol consumption. Methods: We included 373 patients who underwent endoscopy for symptoms, anemia or bleeding investigation. A complete set of demographical, clinical and pathological data was recorded. All patients were successfully genotyped.

Results: In the multivariate logistic regression model, the patients having Pro/Pro genotype of GPX1 gene polymorphism had more severe gastric lesions as compared with patients with the Leu/Pro or Leu/Leu genotype (OR=1.89, 95\%CI: 0.99-3.57, $\mathrm{p}=0.051$ ). The GPX1 Pro198Leu and the MnSOD Ala16Val gene polymorphism could be independent risk factors for reactive gastropathy changes, as shown by their association very close to statistical significance ( $\mathrm{p}=0.059$ and $\mathrm{p}=0.054$, respectively). Consumption of anticoagulants was a significant independent predictor ( $\mathrm{p}=0.023$, OR:0.43 95\%CI:0.21-0.89) for the absence of active gastritis, while low-dose aspirin consumption was a risk factor for active gastritis in biopsy samples ( $\mathrm{p}=0.025$, OR:1.71, 95\%CI:1.07-2.74). Conclusion: The variant genotype of GPX1Pro198Leu was associated with an increased risk for reactive gastropathy changes in gastric biopsies and with less severe endoscopic lesions, while MnSODAla16Val variant genotype (Val/Val or Val/Ala) seems to be related to the reactive gastropathy. However, none of them were associated with inflammatory or premalignant gastric lesions.
\end{abstract}

Key words: CAT-262 C>T - MnSOD Ala16Val - GPX1 Pro198Leu - reactive gastropathy - ulcer.

Abbreviations ACO: anticoagulant; CAT: catalase; gDNA: genomic DNA; GPX1: Glutathione peroxidase 1; GSH: glutathione; H. pylori: Helicobacter pylori; LWMH: low-weight molecular heparin; MnSOD: manganese superoxide dismutase; NSAIDs: non-steroidal anti-inflammatory drugs; PCR-RFLP: polymerase chain reaction and restriction fragment length polymorphism; ROS: reactive oxygen species; SNPs: single nucleotide polymorphisms.

\section{INTRODUCTION}

The current concept of sporadic gastric carcinogenesis admits the Correa cascade model as a multistep multifactorial process with Helicobacter pylori (H. pylori) infection playing a crucial role $[1,2]$, modulated by individual and environmental factors [3]. Chronic inflammatory changes in gastric mucosa, gastric atrophy and intestinal metaplasia are acknowledged steps in the process of gastric carcinogenesis [1]. Oxidative stress is caused by an imbalance between the production of reactive oxygen species (ROS) and the ability to detoxify the reactive intermediates or to repair the resulting damage [4]. The acute and chronic inflammation induced by $H$. pylori causes an increased production of cytokines, the ROS and reactive nitrogen species that are mutagenic and carcinogenic to the gastric mucosa [5]. The increased oxidative stress may lead to cell damage and alterations in the mechanisms that control cell proliferation and apoptosis, which may eventually lead to gastro-duodenal ulcers and gastric cancer. It is also accepted that ROS are also produced in response to cigarette smoking, alcohol consumption, ingestion of non-steroidal anti-inflammatory drugs (NSAIDs) and many other exogenous agents [5]. 
Catalase (CAT) is a heme enzyme that plays important roles in regulating the level of ROS in humans by converting $\mathrm{H}_{2} \mathrm{O}_{2}$ into $\mathrm{H}_{2} \mathrm{O}$ and $\mathrm{O}_{2}$. Single nucleotide polymorphisms (SNPs) have been identified in the promoter regions of the CAT $(-262 \mathrm{C}>\mathrm{T})$, which alter the expression levels of these genes and thus increase the levels of ROS [6,7].

Reactive oxygen species can act by modifying the redox regulation of glutathione (GSH), an important antioxidant. Reduced GSH levels are higher in the mucosa of the stomach in comparison with other regions of the digestive tract, providing additional protection against gastric acid $[5,8]$. Glutathione peroxidase 1 (GPX1) enzyme plays a critical role in the detoxification system, and a SNP of the GPX1 gene, namely Pro198Leu, has been shown to be associated with gastrointestinal cancer in relationship with exposure at various environmental factors $[9,10]$.

The manganese superoxide dismutase (MnSOD) is essential for life as a major antioxidant functioning against ROS or free radicals in the human mitochondria [11]. The MnSOD gene is a nuclear gene encoding this mitochondrial protein and its polymorphism (Val16Ala) was studied in relation with various types of cancers [12]. Some studies revealed that the Ala form of $\mathrm{MnSOD}$ was $30 \%$ to $40 \%$ more efficiently localized to the mitochondria than the Val form, while the Val form is likely to be associated with higher levels of ROS [13], increasing the risk for cancer, including gastric cancer, but results were discordant $[12,14]$.

The aim of our study was to investigate the association between genetic polymorphisms of genes encoding the antioxidative enzymes CAT, GPX and SOD and the presence of endoscopic and histologic gastric lesions, considering also the environmental risk factors such as $H$. pylori infection, drug exposure, smoking, alcohol consumption.

\section{MATERIAL AND METHOD}

\section{Patients}

Patients referred for upper digestive endoscopy between 2014-2015 for dyspeptic symptoms, anemia or for gastrointestinal bleeding risk evaluation were included. Clinical and demographical data were collected using interviews, clinical examinations and medical records. We considered patients consuming at least 10 units $(10 \mathrm{ml})$ of pure alcohol weekly as drinkers and non-exposure as any amount of alcohol consumption below this level. Patients smoking more than 5 cigarettes/day, including quitters during the past 5 years were considered as smokers. We considered gastrotoxic drug exposure, NSAIDs consumption on regular daily basis in patients with arthritis or other inflammatory disorders who required chronic therapy (more than six weeks). Long-term antiplatelet therapy (low-dose aspirin 75$325 \mathrm{mg} /$ day, clopidogrelum 75mg/day more than 6 months) was also considered as gastrotoxic medication. Patients on acenocumarolum and low-weight molecular heparin (LWMH) therapy as daily administration of a regular dose for at least two weeks prior to endoscopy were considered on anticoagulant (ACO) therapy. We excluded the patients with acute bleeding episodes, previous therapy for eradication of $H$. pylori infection, gastric surgery, gastric or esophageal cancer, esophageal varices, severe medical conditions (cancer, cirrhosis, severe heart or renal failure, etc.) and patients with insufficient data (social habits, drug exposure). We had less than $2 \%$ missing histological results, so that our final results could not be influenced.

The Ethical Committee of the University of Medicine and Pharmacy of Tirgu Mures, Romania approved the study $(83 / 22.09 .2014)$ and a written informed consent was obtained from all subjects included.

\section{Endoscopy}

Endoscopy was performed in all patients by an endoscopist blinded to drug exposure. Mucosal lesions were described as erythema, petechiae, erosions, or ulcers. Petechiae were defined as hemorrhagic areas with no mucosal defect and erosions as mucosal defects smaller than $5 \mathrm{~mm}$. Defects larger than $5 \mathrm{~mm}$ in diameter, extended into the deeper layers of the gastric or duodenal wall were defined as ulcers. We used a modified Lanza score [15] to assess gastro-duodenal lesions. For no mucosal lesions a 0 score was given, while for one erosion or petechia a score of 1 was attributed. We considered a score 2 when $2-10$ erosions or submucosal hemorrhages were counted, score 3 for more than 10 erosions or submucosal hemorrhages and score 4 when an ulcer was present (defect larger than $5 \mathrm{~mm}$ ).

Two biopsy specimens from the antrum and two from the corpus (from the lesser and the greater curvature) were obtained for routine histology in every patient and examined by two pathologists also blinded to patient drug exposure and symptoms.

\section{Histology}

The biopsies were examined for routine investigation. Biopsy specimens were fixed in $10 \%$ buffered formalin, routinely processed, embedded in paraffin and stained with hematoxylineosin, PAS-alcian blue and Giemsa. H. pylori infection was considered negative if it was absent from all biopsy sites, and positive if at least one histology test was positive. The degree of mucosal chronic inflammation, activity, H. pylori infection, glandular atrophy and intestinal metaplasia were classified into 4 grades according to the Updated Sydney System. Patients without important inflammation, but with prominent mucin depletion, foveolar hyperplasia, fibro-muscular replacement of the lamina propria, and congestion of the superficial mucosal capillaries were diagnosed as reactive gastropathy [16]. Dominant epithelial changes, but not vascular pathology were the main reactive gastropathy changes in our series; the etiological factors were not investigated in the present research.

\section{Genotyping}

Genomic DNA (gDNA) isolated from fresh blood samples obtained from consecutive patients which underwent upper digestive endoscopies was used for genotyping of CAT -262 C>T, GPX1 Pro198Leu and MnSOD Ala16Val polymorphisms by using polymerase chain reaction and restriction fragment length polymorphism (PCR-RFLP) assays with minor changes as previously reported [17-20]. We performed an internal quality control to avoid errors of genotyping methods used, and randomly DNA samples were re-genotyped and the results were similar to those originally obtained. 


\section{Statistical analysis}

The quantitative characteristics of the patients were described as mean \pm standard deviation (SD), while the qualitative nominal or ordinal characteristics were summarized using absolute and relative frequencies. The significant differences regarding age and gender distributions in the patients' groups were tested by the Student- $t$ and Chi-square tests.

The possible relationships between studied gene polymorphisms and dependent dichotomous variables (endoscopic lesions, premalignant gastric lesions, reactive gastropathy, active gastritis, inactive chronic gastritis) were modeled by logistic regression analysis. The univariate logistic regression highlighted the influence of the studied independent factor on the criterion variable, while multivariate logistic regression identified independent predictors of different type of gastric lesions.

In order to quantify the strength of association, both crude and adjusted odds ratios (OR) were estimated in order to demonstrate the patients' susceptibility of endoscopic and histological gastric lesions. Adjusted ORs from a multivariable model were estimated accounting for demographic variables (such as age and gender) and for known environmental factors such as alcohol and smoking. All models tested in logistic regression were additive models (we also tested the significance of the interaction term between each genetic and environmental factor but interaction terms were not significant).

We did not use the Bonferroni correction in our research due to particularities of the studied variables: three types of histologic gastric changes, considered to be three distinct events of interest, and three independent SNPs were analyzed $[21,22]$.

For all inferential statistical tests, we used the two-sided approach, statistical significance being achieved if estimated significance level was lower than the significance threshold $(\alpha=0.05)$.

The statistical analysis was released with the advanced environment for statistical computing and graphics R (v.3.4.0, Vienna, Austria).

\section{RESULTS}

\section{Patient characteristics}

A number of 373 patients who met the inclusion criteria were enrolled. The mean age \pm SD was $62 \pm 12.7$ years. We analyzed the potential effect of exposures to the variables of interest (genetic and environmental factors), the endoscopic gastro-duodenal lesions occurrence and gastric histological changes. This study comprises, as outcome nominal variables, endoscopic lesions (severe lesions-Lanza score 3 and 4, and mild lesion-Lanza score 1 and 2), and histological changes (premalignant gastric lesions - gastric atrophy and intestinal metaplasia, presence/absence, inactive chronic gastritis presence/absence, active gastritis - presence/absence, reactive gastropathy - presence/absence).

The distribution of the demographic characteristics, genotype of investigated SNPs and environmental factors in patients grouped according to the presence of endoscopic and histological lesions are presented in Tables I and II.
Table I. The distribution of demographic, genetic and environmental factors according to the endoscopic lesion

\begin{tabular}{|c|c|c|c|}
\hline \multicolumn{2}{|c|}{ Variables independent/outcome } & \multicolumn{2}{|c|}{ Lanza Score } \\
\hline & & $\begin{array}{l}\text { Severe } \\
(n=64)\end{array}$ & $\begin{array}{c}\text { Mild } \\
(\mathrm{n}=309)\end{array}$ \\
\hline Age $\left(\right.$ years) ${ }^{*}$ & & $63.9 \pm 10.9$ & $61.6 \pm 13.0$ \\
\hline \multirow[t]{2}{*}{ Gender $^{\dagger}$} & Males & $39(60.9)$ & $124(40.1)$ \\
\hline & Females & $25(39.1)$ & $185(59.9)$ \\
\hline \multirow[t]{2}{*}{$C A T-262 \mathrm{C}>\mathrm{T}^{\dagger}$} & $\mathrm{CT}+\mathrm{TT}$ & $34(53.1)$ & $154(49.8)$ \\
\hline & $\mathrm{CC}$ & $30(46.9)$ & $155(50.2)$ \\
\hline \multirow[t]{2}{*}{ GPX1 Pro198Leu ${ }^{\dagger}$} & Pro/Leu+Leu/Leu & $43(67.2)$ & $247(79.9)$ \\
\hline & Pro/Pro & $21(32.8)$ & $62(20.1)$ \\
\hline \multirow[t]{2}{*}{$M n S O D$ Ala16 $\mathrm{Val}^{\dagger}$} & $\mathrm{Val} / \mathrm{Val}+\mathrm{Val} / \mathrm{Ala}$ & $55(85.9)$ & $275(89.0)$ \\
\hline & Ala/Ala & $9(14.1)$ & $34(11.0)$ \\
\hline \multirow[t]{2}{*}{ Alcohol ${ }^{+* *}$} & Yes & $20(31.2)$ & $73(23.7)$ \\
\hline & No & $44(68.8)$ & $235(76.3)$ \\
\hline \multirow[t]{2}{*}{ Smoking ${ }^{\dagger, \S}$} & Yes & $12(18.8)$ & $46(15.0)$ \\
\hline & No & $52(81.2)$ & $261(85.0)$ \\
\hline \multirow[t]{2}{*}{ Aspirin $^{\dagger}$} & Yes & $37(57.8)$ & $125(40.5)$ \\
\hline & No & $27(42.2)$ & $184(59.5)$ \\
\hline \multirow[t]{2}{*}{$\mathrm{NSAIDs}^{\dagger}$} & Yes & $8(12.5)$ & $38(12.3)$ \\
\hline & No & $56(87.5)$ & $271(87.7)$ \\
\hline \multirow[t]{2}{*}{$\mathrm{ACO}^{\dagger}$} & Yes & $14(21.9)$ & 45 (14.6) \\
\hline & No & $50(78.1)$ & $264(85.4)$ \\
\hline
\end{tabular}

${ }^{*}$ mean $\pm \mathrm{SD} ;{ }^{\dagger}$ number $(\%),{ }^{*}$ consumption of $>10$ units/week, ${ }^{9}>5$ cigarettes/ day including quitters during the past 5 years;NSAIDs: non-steroidal anti-inflammatory drugs, regular daily dose; ACO: anticoagulant therapy (acenocumarolum and low-molecular weight heparin).

There was no significant difference in the age distribution in patients with severe and mild gastro-duodenal lesions (Table I). The mean of age of patients with premalignant lesions or chronic inactive gastritis was significantly higher than the age in patients without these findings $(p<0.00$ and $p=0.013$, respectively). There was no significant difference in the age distribution of patients with or without reactive gastropathy $(p=0.587)$. The same results were obtained for age distribution reported to active gastritis $(\mathrm{p}=0.066)$. Regarding the severity of gastro-duodenal lesions we noticed a higher frequency of males, $60.9 \%$ vs. $39.1 \%$ ( $p=0.002)$ in the subgroup with severe lesions in comparison with the group with mild lesions (Table I). There was no significant difference in repartition by gender in the patients grouped according to the histological findings (premalignant lesions, reactive gastropathy, inactive chronic gastritis and active gastritis $)(\mathrm{p}=0.277, \mathrm{p}=0.130, \mathrm{p}=0.526$ and $\mathrm{p}=0.716$, respectively) (Table II).

Relationship between the gene polymorphisms, environmental factors and severe endoscopic gastroduodenal lesions

On univariate regression analysis, from all studied gene polymorphisms, only GPX1 gene polymorphism was associated with the severity of gastro-duodenal lesions, the presence of a variant genotype showing a protective effect. Aspirin consumption remained an independent risk factor for the severity of gastric lesions in the presence of other covariates (Table III). 
Table II. The distribution of demographic, genetic and environmental factors according to the histological changes in biopsy samples

\begin{tabular}{|c|c|c|c|c|c|c|c|c|c|}
\hline \multicolumn{2}{|c|}{ Variables independent/outcome } & \multicolumn{2}{|c|}{$\begin{array}{c}\text { Gastric premalignant } \\
\text { lesions }\end{array}$} & \multicolumn{2}{|c|}{ Reactive gastropathy } & \multicolumn{2}{|c|}{$\begin{array}{c}\text { Inactive chronic } \\
\text { gastritis }\end{array}$} & \multicolumn{2}{|c|}{ Active gastritis } \\
\hline & & $\begin{array}{l}\text { Present } \\
(n=139)\end{array}$ & $\begin{array}{l}\text { Absent } \\
(\mathrm{n}=227)\end{array}$ & $\begin{array}{l}\text { Present } \\
(\mathrm{n}=130)\end{array}$ & $\begin{array}{l}\text { Absent } \\
(n=236)\end{array}$ & $\begin{array}{l}\text { Present } \\
(\mathrm{n}=85)\end{array}$ & $\begin{array}{l}\text { Absent } \\
(\mathrm{n}=281)\end{array}$ & $\begin{array}{l}\text { Present } \\
(n=133)\end{array}$ & $\begin{array}{l}\text { Absent } \\
(n=233)\end{array}$ \\
\hline \multicolumn{2}{|l|}{ Age (years)* } & $65.2 \pm 11.4$ & $60.4 \pm 13.1$ & $62.5 \pm 12.1$ & $61.7 \pm 12.9$ & $65.2 \pm 11.8$ & $60.9 \pm 12.8$ & $59.8 \pm 13.1$ & $63.3 \pm 12.3$ \\
\hline \multirow[t]{2}{*}{ Gender ${ }^{\dagger}$} & Males & $65(46.8)$ & $93(41.0)$ & $50(38.5)$ & $108(45.8)$ & $37(43.5)$ & $121(43.1)$ & $60(45.1)$ & $98(42.1)$ \\
\hline & Females & $74(53.2)$ & $134(59.0)$ & $80(61.5)$ & $128(54.2)$ & $48(56.5)$ & $160(56.9)$ & $73(54.9)$ & $135(57.9)$ \\
\hline CAT-262 & $\mathrm{CT}+\mathrm{TT}$ & $69(49.6)$ & $114(50.4)$ & $66(51.2)$ & $117(49.6)$ & $40(47.1)$ & $143(51.1)$ & $65(49.2)$ & $118(50.6)$ \\
\hline $\mathrm{C}>\mathrm{T}^{\dagger}$ & $\mathrm{CC}$ & $70(50.4)$ & $112(49.6)$ & $63(48.8)$ & $119(50.4)$ & $45(52.9)$ & $137(48.9)$ & $67(50.8)$ & $115(49.4)$ \\
\hline GPX1 & Pro/Leu++Leu/Leu & $109(78.4)$ & $177(78.0)$ & $109(83.85)$ & $177(75.0)$ & $67(78.8)$ & $219(77.9)$ & $98(73.7)$ & $188(80.7)$ \\
\hline Pro198Leu ${ }^{\dagger}$ & Pro/Pro & $30(21.6)$ & $50(22.0)$ & $21(16.2)$ & $59(25.0)$ & $18(21.2)$ & $62(22.1)$ & $35(26.3)$ & $45(19.3)$ \\
\hline$M n S O D$ & $\mathrm{Val} / \mathrm{Val}+\mathrm{Val} / \mathrm{Ala}$ & $120(86.3)$ & $204(89.9)$ & $121(93.1)$ & $203(86.0)$ & $75(88.2)$ & $249(88.6)$ & $114(85.7)$ & $210(90.1)$ \\
\hline Ala16 Val $^{\dagger}$ & Ala/ala & $19(13.7)$ & $23(10.1)$ & $9(6.9)$ & $33(14.0)$ & $10(11.8)$ & $32(11.4)$ & $19(14.3)$ & $23(9.9)$ \\
\hline \multirow[t]{2}{*}{ Alcohol $^{\dagger, *}$} & Yes & $39(28.1)$ & $50(22.1)$ & $28(21.5)$ & $61(26.0)$ & $18(21.2)$ & $71(25.4)$ & $37(28.0)$ & $52(22.3)$ \\
\hline & No & $100(71.9)$ & $176(77.9)$ & $102(78.5)$ & $174(74.0)$ & $67(78.8)$ & 209 (74.6) & $95(72.0)$ & $181(77.7)$ \\
\hline \multirow[t]{2}{*}{ Smoking ${ }^{\dagger, \S}$} & Yes & $28(20.1)$ & $28(12.4)$ & 15 (11.6) & $41(17.4)$ & $13(15.3)$ & $43(15.4)$ & 25 (1893) & $31(13.4)$ \\
\hline & No & $111(79.9)$ & $197(87.6)$ & $1114(88.4)$ & $194(82.6)$ & $72(84.7)$ & $236(84.6)$ & $107(81.1)$ & $201(86.6)$ \\
\hline \multirow[t]{2}{*}{ Aspirin $^{\dagger}$} & Yes & $66(47.5)$ & $94(41.4)$ & $59(45.4)$ & $101(42.8)$ & $32(37.6)$ & $128(45.6)$ & $66(49.6)$ & $94(40.3)$ \\
\hline & No & $73(52.5)$ & $133(58.6)$ & $71(54.6)$ & $135(57.2)$ & $53(62.4)$ & $153(54.4)$ & $67(50.4)$ & 139 (59.7) \\
\hline \multirow[t]{2}{*}{ NSAIDs $^{\dagger}$} & Yes & $17(12.2)$ & $28(12.3)$ & $15(11.5)$ & $30(12.7)$ & $12(14.1)$ & $33(11.7)$ & $18(13.5)$ & $27(11.6)$ \\
\hline & No & $122(87.8)$ & $199(87.7)$ & $115(88.5)$ & $206(87.3)$ & $73(85.9)$ & $248(88.3)$ & $115(86.5)$ & $206(88.4)$ \\
\hline \multirow[t]{2}{*}{$\mathrm{ACO}^{+}$} & Yes & $27(19.4)$ & $31(13.7)$ & $25(19.2)$ & $33(14.0)$ & $19(22.4)$ & 39 (13.9) & $12(9.0)$ & $46(19.7)$ \\
\hline & No & $112(80.6)$ & $196(86.3)$ & $105(80.8)$ & $203(86.0)$ & $66(77.6)$ & $242(86.1)$ & $121(91.0)$ & $187(80.3)$ \\
\hline
\end{tabular}

${ }^{*}$ arithmetic mean $\pm \mathrm{SD},{ }^{* *}$ number $(\%),{ }^{*}$ consumption of $>10$ units/week; ${ }^{\varsigma}>5$ cigarettes/day including quitters during the past 5 years; NSAIDs: non-steroidal anti-inflammatory drugs, regular daily dose; ACO: anticoagulant therapy (acenocumarolum and low-molecular weight heparin).

Relationship between CAT -262 C>T, GPX1 Pro198Leu and MnSOD Ala16Val gene polymorphisms and the premalignant gastric lesions

The studied gene polymorphisms were not predictors for the presence of premalignant lesions (intestinal metaplasia and gastric atrophy) in our study by regression analysis ( $p>0.05)$. Univariate regression analyses showed a borderline association between smoking and the presence of premalignant gastric lesions ( $\mathrm{p}=0.05, \mathrm{OR}_{\text {crude }}=1.78$, CI 95\%: 1.001-3.15), while multivariate regression analysis revealed no association between the mentioned parameters.

Relationship between CAT -262 C>T, GPX1 Pro198Leu and $M n S O D$ Ala16Val gene polymorphisms and gastric inflammatory and reactive gastropathy changes

Our multivariate regression results showed that the patients with the variant genotype GPX1 Pro198Leu polymorphism had an estimated increased risk to develop

Table III. Logistic regression models: results for endoscopic gastric lesions

\begin{tabular}{|c|c|c|c|c|}
\hline \multirow{3}{*}{$\begin{array}{l}\text { Dependent variables } \\
\text { Independent factors }\end{array}$} & \multicolumn{4}{|c|}{ Lanza Score (Severe vs. Mild) } \\
\hline & \multicolumn{2}{|c|}{ Univariate regression analysis } & \multicolumn{2}{|c|}{ Multivariate regression analysis } \\
\hline & p-value* & $\mathrm{OR}_{\text {crude }}(95 \% \mathrm{CI})$ & p-value ${ }^{*}$ & $\mathrm{OR}_{\text {adjusted }}(95 \% \mathrm{CI} \%)$ \\
\hline$C A T-262 \mathrm{C}>\mathrm{T}(\mathrm{CT}+\mathrm{TT}$ vs. $\mathrm{CC})$ & 0.632 & $1.14(0.67-1.96)$ & 0.322 & $1.34(0.75-2.40)$ \\
\hline GPX1 Pro198Leu (Pro/Leu+Leu/Leu vs. Pro/Pro) & 0.027 & $0.51(0.29-0.93)$ & 0.051 & $0.53(0.28-1.01)$ \\
\hline MnSOD Ala16Val (Val/Val+Val/Ala vs. Ala/Ala) & 0.487 & $0.76(0.34-1.66)$ & 0.531 & $0.76(0.33-1.79)$ \\
\hline H.pylori (positive vs. negative) & 0.221 & $1.42(0.81-2.48)$ & 0.277 & $1.40(0.76-2.57)$ \\
\hline Alcohol $^{\dagger}$ (yes vs. no) & 0.259 & $1.34(0.74-2.42)$ & 0.658 & $0.84(0.39-1.82)$ \\
\hline Smoking ${ }^{*}$ (yes vs. no) & 0.451 & $1.48(0.75-2.95)$ & 0.640 & $1.23(0.52-2.86)$ \\
\hline Aspirin (yes vs. no) & 0.005 & $2.18(1.26-3.77)$ & 0.034 & $1.90(1.05-3.44)$ \\
\hline NSAIDs (yes vs. no) & 0.906 & $1.05(0.46-2.38)$ & 0.582 & $1.28(0.53-3.08)$ \\
\hline ACO (yes vs. no) & 0.148 & $1.64(0.84-3.22)$ & 0.338 & $1.45(0.68-3.07)$ \\
\hline
\end{tabular}


reactive gastropathy, but no statistical significance was found (Table IV).

The MnSOD Ala16Val gene polymorphism was a univariate predictor for the presence of reactive gastropathy, with an increased adjusted risk for reactive gastropathy in patients carrying the variant $M n S O D$ Ala16Val genotype, without reaching a statistical significance (Table IV).

The consumption of anticoagulants was statistically associated with active gastritis $(\mathrm{p}=0.008)$ and it was a significant independent predictor ( $\mathrm{p}=0.023)$ with a negative effect on the susceptibility of active gastritis $(\mathrm{OR}=0.43$, CI 95\%: 0.21-0.89).

\section{DISCUSSION}

It is widely accepted today that gastro-duodenal ulcers and gastric malignancies arise in part from oxidative stress [5]. The increased oxidative stress as a consequence of $H$. pylori infection generates a harmful microenvironment for the host rather than an effective means to eliminate the pathogen, that further may lead to cell damage and a poor control in cell proliferation and apoptosis [23]. Reactive oxygen species were also proved to be produced in response to cigarette smoking, alcohol consumption, ingestion of various drugs as NSAIDs, and many other exogenous agents [6]. There are very few published studies investigating the possible influence of genetic polymorphisms related to the oxidative stress on gastro-duodenal diseases. On the other hand, our previous works showed different influence of various genetic factors on gastroduodenal diseases in comparison with those reported in other populations $[24,25]$. For studying the CAT-262C $>\mathrm{T}$, GPX1 Pro198Leu, MnSOD Ala16Val polymorphism in relation to endoscopic and histologic gastric lesions occurrence [26-28] we investigated the impact of variants of the mentioned SNPs and environmental factors (drug consumptions, alcohol and smoking habits) on endoscopic gastro-duodenal lesions and gastric histological changes in one single multivariable model.

Although the antioxidant enzymes activities such as GPX, SOD and CAT were found to be related with the malignant phenotype in gastrointestinal cancers $[29,30]$, none of them were associated with premalignant gastric lesions in our study. The GPX1 Pro198Leu polymorphism appeared to be associated with an increased risk for endoscopic and histologic changes in gastric mucosa. For the GPX1 Pro198Leu SNP, the aminoacid substitution has been showed to decrease the enzymatic activity of glutathione peroxidase in the cells expressing the mutant protein, which further can increase the oxidative damage [7, 31]. The presence of the variant Leu allele showed a protective effect on gastric mucosal lesions in our population, at the same

Table IV. Logistic regression models: results for reactive gastropathy and gastric inflammation

\begin{tabular}{|c|c|c|c|c|c|c|}
\hline \multirow{2}{*}{$\begin{array}{l}\text { Dependent } \\
\text { variables }\end{array}$} & \multicolumn{2}{|c|}{ Reactive gastropathy } & \multicolumn{2}{|c|}{ Inactive chronic gastritis } & \multicolumn{2}{|c|}{ Active gastritis } \\
\hline & $\begin{array}{c}\text { Univariate } \\
\text { regression analysis }\end{array}$ & $\begin{array}{l}\text { Multivariate } \\
\text { regression analysis }\end{array}$ & $\begin{array}{c}\text { Univariate } \\
\text { regression analysis }\end{array}$ & $\begin{array}{l}\text { Multivariate } \\
\text { regression analysis }\end{array}$ & $\begin{array}{l}\text { Univariate } \\
\text { regression analysis }\end{array}$ & $\begin{array}{c}\text { Multivariate } \\
\text { regression analysis }\end{array}$ \\
\hline
\end{tabular}

\begin{tabular}{|c|c|c|c|c|c|c|c|c|c|c|c|c|}
\hline $\begin{array}{l}\text { Independent } \\
\text { factors }\end{array}$ & $\begin{array}{c}\text { p- } \\
\text { value }^{*}\end{array}$ & $\begin{array}{l}\text { OR } \\
(95 \% \mathrm{CI})\end{array}$ & $\begin{array}{c}\text { p- } \\
\text { value }^{*}\end{array}$ & $\begin{array}{l}\text { OR } \\
(95 \% \mathrm{CI})\end{array}$ & $\begin{array}{c}\text { p- } \\
\text { value }^{*}\end{array}$ & $\begin{array}{l}\text { OR }_{\text {crude }} \\
(95 \% \mathrm{CI})\end{array}$ & $\begin{array}{c}\mathrm{p}- \\
\text { value }^{*}\end{array}$ & $\begin{array}{l}\text { OR } \\
(95 \% \mathrm{CI})\end{array}$ & $\begin{array}{c}\mathrm{p}- \\
\text { value }^{*}\end{array}$ & $\begin{array}{l}\mathrm{OR}_{\text {crude }} \\
(\mathrm{CI} 95 \%)\end{array}$ & $\begin{array}{c}\mathrm{p}- \\
\text { value }^{*}\end{array}$ & $\begin{array}{l}\text { OR }_{\text {adjusted }} \\
95 \% \mathrm{CI})\end{array}$ \\
\hline $\begin{array}{l}C A T-262 \mathrm{C}>\mathrm{T} \\
(\mathrm{CT}+\mathrm{TT} \text { vs. } \mathrm{CC})\end{array}$ & 0.772 & $\begin{array}{c}1.07 \\
(0.69- \\
1.64)\end{array}$ & 0.928 & $\begin{array}{c}0.98 \\
(0.63- \\
1.53)\end{array}$ & 0.517 & $\begin{array}{c}0.85 \\
(0.52- \\
1.39)\end{array}$ & 0.671 & $\begin{array}{c}0.90 \\
(0.54-1.49)\end{array}$ & 0.797 & $\begin{array}{c}0.95 \\
(0.62-1.45)\end{array}$ & 0.882 & $\begin{array}{c}0.97 \\
(0.62- \\
1.52)\end{array}$ \\
\hline $\begin{array}{l}\text { GPX1 Pro198Leu } \\
\text { (Pro/Leu+Leu/Leu } \\
\text { vs. Pro/Pro) }\end{array}$ & 0.052 & $\begin{array}{c}1.73 \\
(1.00- \\
3.01)\end{array}$ & 0.059 & $\begin{array}{c}1.73 \\
(0.98- \\
3.04)\end{array}$ & 0.862 & $\begin{array}{c}1.05 \\
(0.58- \\
1.90)\end{array}$ & 0.734 & $\begin{array}{c}1.11 \\
(0.60-2.06)\end{array}$ & 0.120 & $\begin{array}{c}0.67 \\
(0.41-1.11)\end{array}$ & 0.092 & $\begin{array}{c}0.63 \\
(0.37- \\
1.08)\end{array}$ \\
\hline $\begin{array}{l}\text { MnSODAla16Val } \\
\text { (Val/ Val+Val/ Ala } \\
\text { vs. Ala/Ala) }\end{array}$ & 0.047 & $\begin{array}{c}2.19 \\
(1.01- \\
4.72)\end{array}$ & 0.054 & $\begin{array}{c}2.17 \\
(0.99- \\
4.77)\end{array}$ & 0.924 & $\begin{array}{c}0.96 \\
(0.45- \\
2.05)\end{array}$ & 0.821 & $\begin{array}{c}1.10 \\
(0.50-2.40)\end{array}$ & 0.205 & $\begin{array}{c}0.66 \\
(0.34-1.26)\end{array}$ & 0.134 & $\begin{array}{c}0.59 \\
(0.30- \\
1.17)\end{array}$ \\
\hline $\begin{array}{l}\text { Alcohol }^{\dagger} \\
\text { (Yes vs. No) }\end{array}$ & 0.347 & $\begin{array}{c}0.78 \\
(0.47- \\
1.30)\end{array}$ & 0.916 & $\begin{array}{c}1.04 \\
(0.54- \\
1.98)\end{array}$ & 0.432 & $\begin{array}{c}0.79 \\
(0.44- \\
1.42)\end{array}$ & 0.448 & $\begin{array}{c}0.75 \\
(0.35-1.59)\end{array}$ & 0.223 & $\begin{array}{c}1.36 \\
(0.83-2.21)\end{array}$ & 0.618 & $\begin{array}{c}1.18 \\
(0.62- \\
2.21)\end{array}$ \\
\hline $\begin{array}{l}\text { Smoking } \\
\text { (Yes vs. No) }\end{array}$ & 0.144 & $\begin{array}{c}0.62 \\
(0.33- \\
1.18)\end{array}$ & 0.385 & $\begin{array}{c}0.72 \\
(0.34- \\
1.52)\end{array}$ & 0.979 & $\begin{array}{c}0.99 \\
(0.51- \\
1.95)\end{array}$ & 0.752 & $\begin{array}{c}1.14 \\
(0.50-2.60)\end{array}$ & 0.158 & $\begin{array}{c}1.52 \\
(0.85-2.70)\end{array}$ & 0.414 & $\begin{array}{c}1.34 \\
(0.66- \\
2.71)\end{array}$ \\
\hline $\begin{array}{l}\text { Aspirin } \\
\text { (Yes vs. No) }\end{array}$ & 0.663 & $\begin{array}{c}1.11 \\
(0.72- \\
1.71)\end{array}$ & 0.599 & $\begin{array}{c}1.13 \\
(0.72- \\
1.79)\end{array}$ & 0.199 & $\begin{array}{c}0.72 \\
(0.44- \\
1.19)\end{array}$ & 0.068 & $\begin{array}{c}0.61(0.36- \\
1.04)\end{array}$ & 0.086 & $\begin{array}{l}1.46(0.95- \\
2.24)\end{array}$ & 0.025 & $\begin{array}{c}1.71 \\
(1.07- \\
2.74)\end{array}$ \\
\hline $\begin{array}{l}\text { NSAIDs } \\
\text { (Yes vs. No) }\end{array}$ & 0.744 & $\begin{array}{c}0.90 \\
(0.46- \\
1.73)\end{array}$ & 0.929 & $\begin{array}{c}1.03(0.52- \\
2.06)\end{array}$ & 0.560 & $\begin{array}{c}1.24 \\
(0.61- \\
2.51)\end{array}$ & 0.407 & $\begin{array}{c}1.37(0.65- \\
2.88)\end{array}$ & 0.586 & $\begin{array}{c}1.19(0.63- \\
2.26)\end{array}$ & 0.984 & $\begin{array}{c}1.01 \\
(0.51- \\
1.99)\end{array}$ \\
\hline ACO (Yes vs. No) & 0.190 & $\begin{array}{l}1.47 \\
(0.83- \\
2.59)\end{array}$ & 0.288 & $\begin{array}{c}1.39(0.76- \\
2.54)\end{array}$ & 0.063 & $\begin{array}{c}1.79 \\
(0.97- \\
3.30)\end{array}$ & 0.236 & $\begin{array}{c}1.48(0.78- \\
2.82)\end{array}$ & 0.008 & $\begin{array}{l}0.40(0.21- \\
0.79)\end{array}$ & 0.023 & $\begin{array}{c}0.43 \\
(0.21- \\
0.89)\end{array}$ \\
\hline
\end{tabular}

${ }^{\star}$ Obtained from Wald's test, bold estimated values for p-value denoted statistical significance while italic values denoted a tendency toward statistical signification $(\mathrm{p}<0.10)$; OR from multivariable model were also adjusted for age and gender. $\dagger$ consumption of $>10$ units/week $\ddagger>5$ cigarettes/day including quitters during the past 5 years; NSAIDs: non-steroidal anti-inflammatory drugs, regular daily dose; ACO: anticoagulant therapy (acenocumarolum and low-molecular weight heparin) 
time increasing the risk for occurrence of reactive gastropathy changes. The borderline negative correlation with active gastritis and the lack of relation with premalignant lesions suggest a possible beneficial effect of the variant genotype to sustain the innate and adaptive host immune response against the most important aggressor of gastric mucosa, $H$. pylori infection, which can lead to ulcer and cancer occurrence.

The GPX1 Pro198Leu polymorphism seemed in our study group to decrease the inflammatory response and limit the progress toward mucosal injury, but to increase the risk for reactive gastropathy as a mucosa response to chemical aggressors (bile reflux, drugs, ethanol). The GPX1 Pro198Leu polymorphism might modulate the chronic oxidative stress produced by gastric cells as a response to both, chemical aggressor and $H$. pylori infection, in a different way. The GPX1 Pro198Leu polymorphism plays a different role in gastric endoscopic and histological changes among different ethnic populations, as gastric diseases are complex, while different genetic background may contribute, in combination with environmental factors, to their development $[7,11,14]$.

It was reported that reduced SOD activity causes gastric ulcers, and increased SOD activity is associated with ulcer healing [32], while gastric adenocarcinoma tissues exhibit increased expression of MnSOD compared to the normal mucosa [33]. Our study proved an association between reactive gastropathy and $\mathrm{MnSOD}$ Ala16Val gene polymorphism. In addition, it seemed to be an independent risk factor for reactive gastropathy in gastric biopsies after adjusting for other clinical and environmental factors, the patients with the Val/Val or Ala/Val genotype having more than a twofold increase in odds of reactive gastropathy in gastric biopsies. The complex gene-environment and gene-gene interplay could explain our findings, as Tu et al. [28] sustained that carriers of the Ala allele have an increased risk for dysplasia modulated by $H$. pylori infection in gastric mucosa, which may be due to a higher activity form of enzyme. In our population, with a high prevalence of $H$. pylori infection, the results sustain the possible role of $\mathrm{MnSOD}$ Ala16Val polymorphism in increasing the risk for reactive gastropathy changes as a response against chemical aggressors, maybe as an effect of imbalance between ROS generation and oxidative defense. It has been shown that the $M n S O D$ Ala16Val polymorphism could modulate the effect of exogenous antioxidants against cancer $[28,34]$.

The CAT -262C>T gene polymorphism seems not to modulate the gastric lesions occurrence in our study, despite the previous observations which have shown that less catalase activity is correlated with gastric adenocarcinoma, and $H$. pylori-gastritis [35]. Our findings are similar to those reported by Steenport et al. [26], which indicated that the CAT $-262 \mathrm{C}>\mathrm{T}$ SNP was not associated with an individual's susceptibility to developing precancerous lesions such as gastric atrophy or intestinal metaplasia.

We investigated also the influence of CAT $-262 \mathrm{C}>\mathrm{T}, \mathrm{GPX} 1$ Pro198Leu, MnSOD Ala16Val gene polymorphisms, and of some clinical and environmental factors $(H$. pylori, alcohol, smoking, aspirin, NSAIDs, ACO) on premalignant gastric lesion occurrence. From all environmental factors investigated, only smoking was associated with premalignant gastric lesions, sustaining the role of smoking as an independent risk factor for premalignant lesions and gastric cancer [36, 37], as well as for mucosal defects as shown in our previous study [38].

Another surprising result of our study was the protective effect of the ACO therapy for active inflammatory changes in gastric mucosa obtained after adjustment with other concomitant possible factors. Although a "test and treat $H$. pylori infection" strategy before starting ACOs was not applied in our population, a selection bias or possible pathological mechanism could explain the findings. This unexpected result should be further investigated in larger studies and settings. At the same time, aspirin consumption was borderline negatively associated with inactive chronic gastritis and increased the risk for active gastritis. These observations support the synergistic polymorphonuclearmediated mechanism of gastric mucosal injury by aspirin and $H$. pylori infection [39] in populations with a high prevalence of infection with specific clinical, environmental and genetic risk factors influencing the gastric lesions in the presence of both aggressors [40, 41]. The different findings observed in our study may be explained by the diversity of genetic, ethnic, lifestyle and environmental background of the population investigated. The importance of our investigation is sustained by recent researches supporting that genetic and environmental factors, as well as alterations in gastric cell adaptive mechanisms to oxidative stress, are the crucial factors involved in gastric diseases [42].

The limitations of our study were the lack of randomization of the sample and the absence of external validation of the logistic models due to the relatively small sample size with patients coming from a single geographic area. Our study might not have sufficient power to identify an association between CAT -262C>T, GPX1 Pro198Leu, MnSOD Ala16Val gene polymorphisms and premalignant gastric lesions, because many SNPs or environmental factors interaction are involved in the progression of gastric cancer. It revealed, surprisingly, the association between GPX1 Pro198Leu, MnSOD Ala16Val gene polymorphism and reactive and inflammatory histologic changes.

The strength of our study is the concomitant evaluation of the endoscopic and histologic features and the three gene polymorphisms involved in the defense against oxidative stress in gastric mucosa. To the best of our knowledge, this is the first study that investigated the multiple genetic potential predictors implicated in oxidative stress and gastric inflammation, reactive gastropathy, premalignant lesions and mucosal defects. In addition, this is the first study performed on a European population which investigates the association of CAT $-262 \mathrm{C}>\mathrm{T}$ gene polymorphism with gastric lesions.

\section{CONCLUSIONS}

Among the three gene polymorphisms investigated, namely CAT -262C>T, GPX1 Pro198Leu, MnSOD Ala16Val, the variant genotype of GPX1 Pro198Leu (Pro/Leu or Leu/Leu) was associated with reactive gastropathy changes in gastric biopsies and less severe endoscopic lesions, while $M n S O D$ Ala16Val variant genotype (Val/Val or Val/Ala) seems to be associated with reactive gastropathy changes in gastric biopsies in the studied population. 
Conflicts of interest: None to declare.

Authors' contributions: A.N. and C.B.: study concept and design, A.N., S.M., F.T. and A.C.: acquisition of data, A.N.: endoscopic examinations; C.B., F.T. and A.C.: genetic study; M.I: statistical analysis and interpretation of data; A.N., M.I., C.B.: drafting of the manuscript; S.M.: histological analysis; All authors approved the final draft submitted.

Acknowledgement: This work was partly supported by a grant from the Romanian National Authority for Scientific Research and Innovation, CNCS/CCCDI-UEFISCDI, project number PN-III-P22.1-PED-2016-1076 within PNCDI III, contract no.147 PED/2017 and by an intern research grant from the University of Medicine and Pharmacy, Tirgu Mures, Romania (Nr. 11/23.12.2014).

\section{REFERENCES}

1. Rugge M, Genta RM, Graham DY, et al. Chronicles of a cancer foretold: 35 years of gastric cancer risk assessment. Gut 2016;65:721-725. doi:10.1136/ gutjnl-2015-310846

2. Correa P. A human model of gastric carcinogenesis. Cancer Res 1988;48:35543360 .

3. Venerito M, Vasapolli R, Rokkas T, Malfertheiner P. Helicobacter pylori and Gastrointestinal Malignancies. Helicobacter 2015;20 Suppl 1:36-39. doi:10.1111/ hel. 12255

4. Mena S, Ortega A, Estrela JM. Oxidative stress in environmental-induced carcinogenesis. Mutat Res 2009;674:36-44. doi:10.1016/j.mrgentox.2008.09.017

5. Bhattacharyya A, Chattopadhyay R, Mitra S, Crowe SE. Oxidative Stress: An Essential Factor in the Pathogenesis of Gastrointestinal Mucosal Diseases. Physiol Rev 2014;94:329-354. doi:10.1152/physrev.00040.2012

6. Forsberg L, Lyrenas U, De Faire R and Mortgenstern A. A common functional C-T substitution in the promoter region of the human catalase gene influences transcription factor binding, reporter gene transcription and is correlated to blood catalase levels. Free Radical Biol Med 2001;30:500-505. doi:10.1016/ S0891-5849(00)00487-1

7. Ji M, Tang J, Zhao J, Xu B, Qin J, Lu J. Polymorphisms in genes involved in drug detoxification and clinical outcomes of anthracycline-based neoadjuvant chemotherapy in Chinese Han breast cancer patients. Cancer Biol Ther 2012;13:264-271. doi:10.4161/cbt.18920

8. Naito Y, Yoshikawa T. Molecular and cellular mechanisms involved in Helicobacter pylori-induced inflammation and oxidative stress. Free Radic Biol Med 2002;33:323-336. doi:10.1016/\$0891-5849(02)00868-7

9. Baroudi O, Benammar-Elgaaied A. Involvement of genetic factors and lifestyle on the occurrence of colorectal and gastric cancer. Crit Rev Oncol Hematol 2016;107:72-81. doi:10.1016/j.critrevonc.2016.08.014

10. Pérez S, Taléns-Visconti R, Rius-Pérez S, Finamor I, Sastre J. Redox signaling in the gastrointestinal tract. Free Radic Biol Med 2017;104:75-103. doi:10.1016/j. freeradbiomed.2016.12.048

11. Attatippaholkun W, Wikainapakul K. Predominant genotypes and alleles of two functional polymorphisms in the manganese superoxide dismutase gene are not associated with Thai cervical or breast cancer. Asian Pac J Cancer Prev 2013;14:3955-3961. doi:10.7314/APJCP.2013.14.6.3955

12. Bag A, Bag N. Target sequence polymorphism of human manganese superoxide dismutase gene and its association with cancer risk: a review. Cancer Epidemiol Biomarkers Prev 2008;17:3298-3305. doi:10.1158/1055-9965.EPI-08-0235
13. Sutton A, Khoury H, Prip-Buus C, Cepanec C, Pessayre D, Degoul F. The Ala16Val genetic dimorphism modulates the import of human manganese superoxide dismutase into rat liver mitochondria. Pharmacogenetics 2003;13:145-157.

14. Moradi MT, Yari K, Rahimi Z, Kazemi E, Shahbazi M. Manganese superoxide dismutase (MnSOD Val-9Ala) gene polymorphism and susceptibility to gastric cancer. Asian Pac J Cancer Prev 2015;16:485488. doi:10.7314/APJCP.2015.16.2.485

15. Lanza FL. Endoscopic studies of gastric and duodenal injury after the use of ibuprofen, aspirin and other NSAIDs. Am J Med 1984;77:19-24. doi:10.1016/S0002-9343(84)80014-5

16. Genta RM. Differential diagnosis of reactive gastropathy. Semin Diagn Pathol 2005;22:273-283. doi:10.1053/j.semdp.2006.04.001

17. Zhang Y, Zhang L, Sun D, Li Z, Wang L, Liu P. Genetic polymorphisms of superoxide dismutases, catalase, and glutathione peroxidase in agerelated cataract. Mol Vis 2011;17:2325-2332.

18. Suzen HS, Gucyener E, Sakalli O, et al. CAT C-262T and GPX1 Pro198Leu polymorphisms in a Turkish population. Mol Biol Rep 2010;37:87-92. doi:10.1007/s11033-009-9540-4

19. Manica-Cattani MF, Cadoná FC, de OliveiraR, et al. Impact of obesity and Ala16Val MnSOD polymorphism interaction on lipid, inflammatory and oxidative blood biomarkers. OJGen 2012;2:202-209. doi:10.4236/ojgen.2012.24026

20. Bănescu C, Iancu M, Trifa AP, et al. From Six Gene Polymorphisms of the Antioxidant System, Only GPX Pro198Leu and GSTP1 Ile105Val Modulate the Risk of Acute Myeloid Leukemia. Oxid Med Cell Longev 2016;2016:2536705. doi:10.1155/2016/2536705

21. Ranstam J. Multiple P-values and Bonferroni correction. Osteoarthritis Cartilage 2016;24:763-764. doi:10.1016/j.joca.2016.01.008

22. Bland JM, Altman DG. Multiple significance tests: the Bonferroni method. BMJ 1995;310:170. doi:10.1136/bmj.310.6973.170

23. Butcher LD, den Hartog G, Ernst PB, Crowe SE. Oxidative Stress Resulting From Helicobacter pylori Infection Contributes to Gastric Carcinogenesis. Cell Mol Gastroenterol Hepatol 2017;3:316-322. doi:10.1016/j.jcmgh.2017.02.002

24. Negovan A, Iancu M, Moldovan V, Mocan S, Banescu C. The Interaction between GSTT1, GSTM1, and GSTP1 Ile105Val Gene Polymorphisms and Environmental Risk Factors in Premalignant Gastric Lesions Risk. Biomed Res Int 2017;2017:7365080. doi:10.1155/2017/7365080

25. Negovan A, Iancu M, Moldovan V, et al. Influence of MDR1 C3435T, CYP2C19*2 and CYP2C19*3 gene polymorphisms and clinical characteristics on the severity of gastric lesions: a case-control study. Gastrointestin Liver Dis 2016;25:258-260. doi:10.15403/ jgld.2014.1121.252.mdr

26. Steenport M, Eom H, Uezu M, et al. Association of polymorphisms in myeloperoxidase and catalase genes with precancerous changes in the gastric mucosa of patients at inner-city hospitals in New York. Oncol Rep 2007;18:235-240. doi:10.3892/or.18.1.235

27. Yi JF, Li YM, Liu T, et al. Mn-SOD and CuZn-SOD polymorphisms and interactions with risk factors in gastric cancer. World J Gastroenterol 2010;16:4738-4746. doi:10.3748/wjg.v16.i37.4738

28. Tu HK, Pan KF, Zhang Y, et al. Manganese superoxide dismutase polymorphism and risk of gastric lesions, and its effects on chemoprevention in a Chinese population. Cancer Epidemiol Biomarkers Prev 2010;19:1089-1097. doi:10.1158/1055-9965.EPI-09-1174

29. Kekec Y, Paydas S, Tuli A, Zorludemir S, Sakman G, Seydaoglu G. Antioxidant enzyme levels in cases with gastrointesinal cancer. Eur J Intern Med 2009;20:403-406. doi:10.1016/j.jim.2008.12.003 
30. Kodydková J, Vávrová L, Kocík M, Žák A. Human catalase, its polymorphisms, regulation and changes of its activity in different diseases. Folia Biol (Praha) 2014;60:153-167.

31. Bastaki M, Huen K, Manzanillo P, et al. Genotype-activity relationship for Mn-superoxide dismutase, glutathione peroxidase 1 and catalase in humans. Pharmacogenet Genomics 2006;16:279-286. doi:10.1097/01. fpc.0000199498.08725.9c

32. Naito Y, Yoshikawa T, Ando T, et al. Changes in superoxide dismutase activity in the gastric mucosa of peptic ulcer patients. J Clin Gastroenterol 1992;14:S131-S134.

33. Janssen AM, Bosman CB, van Duijn W, et al. Superoxide dismutases in gastric and esophageal cancer and the prognostic impact in gastric cancer. Clin Cancer Res 2000;6:3183-3192.

34. Mikhak B, Hunter DJ, Spiegelman D, et al. Manganese superoxide dismutase (MnSOD) gene polymorphism, interactions with carotenoid levels and prostate cancer risk. Carcinogenesis 2008;29:2335-2340. doi:10.1093/carcin/bgn212

35. Monari M, Foschi J, Calabrese C, et al. Implications of antioxidant enzymes in human gastric neoplasms. Int J Mol Med 2009;24:693-700. doi:10.3892/ijmm_00000281

36. González CA, Pera G, Agudo A, et al. Smoking and the risk of gastric cancer in the European Prospective Investigation into Cancer and Nutrition (EPIC). Int J Cancer 2003;107:629-634. doi:10.1002/ijc.11426
37. Abangah G, Rahmani A, Hafezi-Ahmadi MR, et al. Precancerous histopathologic lesions of upper gastrointestinal tract among dyspeptic patients upon endoscopic evaluations. J Gastrointest Cancer 2016;47:1 7. doi:10.1007/s12029-015-9760-x

38. Negovan A, Iancu M, Moldovan V, et al. Clinical Risk Factors for Gastroduodenal Ulcer in Romanian Low-Dose Aspirin Consumers. Gastroenterol Res Pract 2016;2016:7230626. doi:10.1155/2016/7230626

39. Leung Ki EL, Chan FK. Interaction of Helicobacter pylori infection and low-dose aspirin in the upper gastrointestinal tract: implications for clinical practice. Best Pract Res Clin Gastroenterol 2012;26:163-172. doi:10.1016/j.bpg.2012.01.006

40. Negovan A, Voidăzan S, Pantea M, et al. AGT A-20C (rs5050) gene polymorphism and ulcer occurrence in patients treated with low-dose aspirin: a case-control study. Rev Romana Med Lab 2015;23:179-187. doi:10.1515/rrlm-2015-0017

41. Hayashi R, Tahara T, Shiroeda H, et al. Association of genetic polymorphisms in IL17A and IL17F with gastro-duodenal diseases. J Gastrointestin Liver Dis 2012;21:243-249.

42. Díaz P, Valenzuela Valderrama M, Bravo J, Quest AFG. Helicobacter pylori and Gastric Cancer: Adaptive Cellular Mechanisms Involved in Disease Progression. Front Microbiol 2018;9:5. doi:10.3389/ fmicb.2018.00005 